\title{
A CONVERGENT FAMILY OF DIFFUSION PROCESSES WHOSE DIFFUSION COEFFICENTS DIVERGE
}

\author{
BY WALTER A. ROSENKRANTZ
}

Communicated by H. Kesten, February 26, 1974

1. Introduction. The problem of deriving suitable hypotheses from which one can conclude the weak convergence of a family of diffusion processes $x_{n}(t, \omega)$ to a limiting diffusion process $x(t, \omega)$ as $n \rightarrow \infty$ has attracted much attention in recent years. One popular approach is to study the asymptotic behavior of the diffusion coefficients. Specifically let us assume that $\left\{x_{n}(t, \omega), 1 \leqq n<+\infty\right\}$ and $x(t, \omega)$ are one-dimensional, strong Markov processes with continuous paths and stationary transition probabilities. Assume further that the infinitesimal generators $G_{n}$ and $G$ of the corresponding semigroups

$$
T_{n}(t) f(x)=E_{x} f\left(x_{n}(t, \omega)\right) \text { and } T(t) f(x)=E_{x} f(x(t, \omega))
$$

are classical second order differential operators of the form:

$$
\begin{aligned}
G_{n} f(x) & =a_{n}(x) f^{\prime \prime}(x)+b_{n}(x) f^{\prime}(x), & & a_{n}(x)>0, \\
G f(x) & =a(x) f^{\prime \prime}(x)+b(x) f^{\prime}(x), & & a(x)>0 .
\end{aligned}
$$

Under sufficiently stringent hypotheses, Skorohod [8], Borovkov [1], Stroock-Varadhan [9], among others, have shown that a condition of the form

$$
\lim _{n \rightarrow \infty} a_{n}(x)=a(x), \quad \lim _{n \rightarrow \infty} b_{n}(x)=b(x)
$$

is sufficient to conclude convergence of the semigroups, i.e.

$$
\lim _{n \rightarrow \infty} T_{n}(t) f(x)=T() f(x)
$$

for all $f$ in a sufficiently large class of functions. It is known however that the infinitesimal generator $G$ of the diffusion process $x(t, \omega)$ need not be a classical second order differential operator but instead can be one

AMS (MOS) subject classifications (1970). Primary 60F05, 60J60; Secondary 60J35, $60 \mathrm{H} 10$.

Key words and phrases. Diffusion processes, weak convergence, generalized second order differential operators, Trotter-Kato theorem.

Copyright (c) American Mathematical Society 1974 
of Feller's generalized second order differential operators of the form

$$
G f(x)=D_{m} D_{p}^{+} f(x)
$$

where $m$ and $p$ are the speed and scale measures of the diffusion process $x(t, \omega)$. Of course every classical operator $G$ can be put into the Feller form (4) via the recipe (see Mandl [6]):

$$
\begin{aligned}
d p(x) & =\exp (-B(x)) d x, \\
d m(x) & =a(x)^{-1} \exp (B(x)) d x, \\
B(x) & =\int^{x} b(y) \cdot a(y)^{-1} d y .
\end{aligned}
$$

The converse is not true. For example if $a(x)>0$ is continuous and $b(x)$ is locally integrable, then $p^{\prime}(x)$ and $m^{\prime}(x)$ exist everywhere and it is very easy to construct $p$ and $m$ for which these derivatives do not exist everywhere. These remarks suggest that it ought to be possible to have a family of diffusion processes $x_{n}(t, \omega)$ converging weakly as $n \rightarrow \infty$ to a limiting diffusion process $x(t, \omega)$ with the following properties: The infinitesimal generators $G_{n}$ are classical operators of the form (1), but the infinitesimal generator $G$ of the limit process is a generalized operator of the form (4) which is not a classical second order differential operator. In part 2 we give a simple, by no means artificial, example of exactly this kind of phenomenon from which we shall deduce the following interesting consequences:

THEOREM 1. Any condition for the weak convergence of diffusion processes based on the asymptotic behavior of the diffusion coefficients is merely sufficient-it is not necessary.

THEOREM 2. The class of diffusion processes with stochastic integral representations of the form

$$
d x(t, \omega)=\sigma(x(t, \omega)) d w(t, \omega)+b(x(t, \omega)) d t
$$

(here $w(t, \omega)$ is the Wiener process) is not closed with respect to weak convergence of stochastic processes.

2. The example. Let $x(t)$ (to simplify the notation we drop the $\omega$ ) be the solution to the stochastic differential equation

$$
d x(t)=d w(t)+b(x(t)) d t
$$

where $b(x)$ is in $L^{1}(-\infty, \infty)$ and $\int_{-\infty}^{\infty} b(x) d x=\alpha$. Let $x_{n}(t)=x\left(n^{2} t\right) / n$. Then $x_{n}(t)$ satisfies the stochastic differential equation

$$
d x_{n}(t)=d w(t)+b_{n}\left(x_{n}(t)\right) d t
$$

where $b_{n}(x)=n b(n x)$. 
The following theorem is proved in the author's paper [7].

THEOREM 3. If $\alpha=0$ then $x_{n}(t)$ converges weakly to the Wiener process as $n \rightarrow \infty$.

If $\alpha \neq 0$ then $x_{n}(t)$ converges weakly to a diffusion process whose infinitesimal generator $G$ has the Feller form $D_{m} D_{p}^{+}$, where $p(x)=c_{1} x$, $x \geqq 0$, and $p(x)=c_{2} x$ if $x \leqq 0, m(x)=2 c_{1}^{-1} x, x \geqq 0$, and $m(x)=2 c_{2}^{-1} x$ if $x \leqq 0$, and $c_{2}=c_{1} \exp (2 \alpha)$-so $\alpha \neq 0$ implies that $c_{1} \neq c_{2}$ and hence $p^{\prime}(0)$ and $m^{\prime}(0)$ do not exist. Clearly the limit process depends on $\alpha$.

REMARK. This is a far reaching generalization of a theorem of GihmanSkorohod to be found in [3, p. 152].

It is obvious from (7) that $\lim _{n \rightarrow \infty}\left|b_{n}(0)\right|=+\infty$ if $b(0) \neq 0$, and, in fact, if $b(x)$ does not vanish at infinity, then $\lim _{n \rightarrow \infty} b_{n}(x)$ need not exist for any $x$. Indeed if $\alpha \neq 0$, there are no diffusion coefficients (in the classical sense) to which $a_{n}(x) \equiv \frac{1}{2}$ and $b_{n}(x)=n b(n x)$ can converge. This establishes the assertion of Theorem 1. It is equally clear that when $\alpha \neq 0, d x(t)$ does not possess a stochastic integral representation, for otherwise its infinitesimal generator would be a classical operator of the type defined at (1).

Our proof of Theorem 3 is based on the simple observation that

$$
\lim _{n \rightarrow \infty} p_{n}(x)=p(x) \text { and } \lim _{n \rightarrow \infty} m_{n}(x)=m(x),
$$

where $m_{n}$ and $p_{n}$ are the speed and scale measures of the $x_{n}(t)$ process. (The reader can easily check this for himself using (5).) From this fact we deduce the convergence of the resolvents, i.e.

$$
\lim _{n \rightarrow \infty}\left\|\left(\lambda-G_{n}\right)^{-1} f-(\lambda-G)^{-1} f\right\|=0
$$

for every $\lambda>0$ and every $f \in C_{0}(R)$, the bounded continuous functions vanishing at infinity.

From the Trotter-Kato theorem (see [10], [11]) we conclude immediately that

$$
\lim _{n \rightarrow \infty} \sup _{0 \leqq t \leqq z}\left|T_{n}(t) f-T(t) f\right|=0 \quad \text { all } f \in C_{0}(R) .
$$

REMARK. This example can also be exploited to show that various conditions due to Trotter [10, Theorem 5.3], Chernoff [2], and Skorohod [8, Theorem 4.6] of the type

$$
\lim _{n \rightarrow \infty} G_{n} f(x)=G f(x)
$$

are sufficient and not necessary. For another example of this phenomenon see a recent paper of Goldstein [4]. Our example also emphasizes the importance of Kurtz' work [5], who does obtain a necessary and sufficient 
condition for (10) to hold in terms of a different notion of convergence. We note, in conclusion, that while there is no generalization of the "Feller form" to higher-dimensional space the reformulation of the problem in terms of convergence of the resolvents suffers from no such defect.

\section{REFERENCES}

1. A. A. Borovkov, Theorems on the convergence to Markov diffusion processes, Z. Wahrscheinlichkeitstheorie und Verw. Gebiete 16 (1970), 47-76. MR 44 \#2277.

2. P. R. Chernoff, Note on product formulas for operator semigroups, J. Functional Analysis 2 (1968), 238-242. MR 37 \#6793.

3. I. I. Gihman and A. V. Skorohod, Stochastic differential equations, "Naukova Dumka", Kiev, 1968; English transl., Ergebnisse der Mathematik und ihrer Grenzgebiete, Band 72, Springer-Verlag, Berlin and New York, 1972. MR 41 \#7777.

4. J. Goldstein, Some counterexamples involving selfadjoint operators, Rocky Mountain J. Math. 2 (1972), 143-149.

5. T. G. Kurtz, Extensions of Trotter's operator semigroup approximation theorems, J. Functional Analysis 3 (1969), 354-375. MR 39 \#3351.

6. P. Mandl, Analytical treatment of one-dimensional Markov processes, Die Grundlehren der math. Wissenschaften, Band 151, Academia, Prague; Springer-Verlag, New York, 1968. MR 40 \#930.

7. W. Rosenkrantz, Limit theorems for solutions to a class of stochastic differential equations, Indiana Univ. Math. J. (to appear).

8. A. V. Skorohod, Limit theorems for Markov processes, Teor. Verojatnost. i Primenen. 3 (1958), 217-264=Theor. Probability Appl. 3 (1958), 202--246. MR 21 \#370.

9. D. W. Stroock and S. R. S. Varadhan, Diffusion processes with continuous coefficients. II, Comm. Pure Appl. Math. 22 (1969), 479-530. MR 40 \#8130.

10. H. Trotter, Approximation of semi-groups of operators, Pacific J. Math. 8 (1958), 887-919. MR 21 \#2190.

11. K. Yosida, Functional analysis, Die Grundlehren der math. Wissenschaften, Band 123, Academic Press, New York; Springer-Verlag, Berlin, 1965. MR 31 \#5054.

Department of Mathematics, University of Massachusetts, Amherst, MassaCHUSETTS 01002 